\title{
MONOIDS OF INTERVALS OF SIMPLE REFINEMENT MONOIDS AND NON-STABLE K-THEORY OF MULTIPLIER ALGEBRAS
}

\author{
F. ORTUS AND E. PARDO
}

Al Marcel Porti Vilaseca

\begin{abstract}
We show that the representation of the monoid of intervals of a simple refinement monoid in terms of affine semicontinuous functions, given by Perera in 2001, fails to be faithful in the case of strictly perforated monoids. We give some potential applications of this result in the context of monoids of intervals and K-Theory of multiplier rings.
\end{abstract}

\section{INTRODUCTION}

Monoids of intervals of partially ordered abelian groups (or of abelian monoids) has been a topic of interest in the last years, usually as a tool to analyze some other algebraic structures. For example, Wehrung used intervals in [30] in order to give a complete description of the universal theory of Tarski's equidecomposability types semigroups. Also, he used intervals in [31] as an instrument to obtain some extensions of Edwards' Separation Theorem (see, e.g. [10, Theorem 11.13]).

A place where intervals played an important role was in the context of $\mathrm{C}^{*}$-algebras. Goodearl and Handelman [11], [12] used intervals to give a complete classification of extensions of AF $\mathrm{C}^{*}$-algebras. Also, Goodearl [13] used intervals in order to describe the ideal structure of the multiplier algebra $\mathcal{M}(A)$ of a $\sigma$-unital, non-unital $\mathrm{C}^{*}$-algebra of real rank zero and stable rank one $A$. In fact, he proved that the group $K_{0}(\mathcal{M}(A))$ is order-isomorphic to the universal group of the monoid of countable generated intervals on $K_{0}^{+}(A)$. Recently, Perera [19] strengthened Goodearl's results by working with monoids of intervals over $V(A)$, in the particular case of a simple $\mathrm{C}^{*}$-algebra $A$ with $V(A)$ strictly unperforated. He showed that $V(\mathcal{M}(A))$ is isomorphic to $V(A) \sqcup W_{\sigma}^{d}\left(S_{u}\right)$, where $W_{\sigma}^{d}\left(S_{u}\right)$ is a semigroup of affine lower semicontinuous functions. These results allowed to study the ideal structure of $V(\mathcal{M}(A))$ and $V(\mathcal{M}(A) / A)$, as well as its cancellation properties, working with functions instead of intervals, which allows to obtain interesting results in a simplest way. A relevant property enjoyed by the $\mathrm{C}^{*}$-algebra $A$ in the above mentioned cases is that $K_{0}(A)$ turns out to be a Riesz group (see, e.g. [10]). This result, proved by Zhang [33, Theorem 3.2], endows $K_{0}(A)$ with an extra structure that plays a major role in the work of Goodearl and Perera, allowing them to obtain important results about the structure of $V(\mathcal{M}(A))$.

Date: February 25, 2014.

1991 Mathematics Subject Classification. Primary 6F20, 20K20; Secondary 16A50, 16D70, 19K14, 46L55.

Key words and phrases. simple refinement monoid, monoid of intervals, multiplier ring.

First author is supported by a FPU fellow of Junta de Andalucía. The research of the second author is partially supported by DGESIC grant BFM2001-3141, by the Comissionat per Universitats i Recerca de la Generalitat de Catalunya. Both authors are partially supported by PAI III grant FQM-298 of Junta de Andalucía. 
The idea of our work is to follow this line, i.e. to abstract the study to monoids of intervals of simple, cancellative, non-atomic, refinement monoids (i.e. to positive cones of simple Riesz groups), and to translate the results we obtain to the K-theoretical context. In this line is essential to consider into our scope recent results of Wehrung [32], and Pardo [15], [18], where some methods for constructing large families of strictly perforated simple Riesz groups are introduced. The examples obtained in these works allow to construct monoids of intervals satisfying special pathologies, such as failure of separativity of the monoid of intervals (see [1]), among others. Also, Villadsen [25], Rørdam and Villadsen [21], Elliott and Villadsen [7], and Toms [22] constructed examples of simple $C^{*}$-algebras of stable rank one whose $K_{0}$ groups are torsion free and strictly perforated. These examples suggests the possibility of constructing $C^{*}$-algebras $A$ with real rank zero, stable rank one, with $K_{0}(A)$ being strictly perforated. Since $K_{0}(A)$ is then a simple Riesz group, the connection between both kinds of examples suggests the convenience of studying the results of Goodearl and Perera in the strictly perforated case, in order to extend the scope of their results to a wider class. Aside of this application, it is interesting to study monoids of intervals in this case, in order to get new pathological examples in the monoid-theoretical context.

In this paper we study the monoids of intervals of non-atomic, simple, strictly perforated monoids, specially in the countable case (that corresponds to separable $\mathrm{C}^{*}$-algebras or countable von Neumann regular rings). We center our interest in Perera's functional representation, and we conclude that under mild hypotheses this representation is always onto. Also, we characterize injectivity of this representation, and as a consequence we obtain a specially interesting failure of strong separativity in a particular subsemigroup of intervals. Unfortunately we haven't been able to characterize failure of separativity of the monoid of intervals in terms of strict perforation.

The paper is organized as follows. Section 1 is devoted to introduce the basic definitions and results needed to develop our task. In Section 2, we study a particular kind of soft interval, that becomes the key point for pointing out our results on Perera's representation. This is done in Section 3, where we outline some special applications of these results. Finally, Section 4 contains the applications to the context of multiplier rings for non-unital $\mathrm{C}^{*}$-algebras and von Neumann regular rings.

Throughout, we will refer to [10] for the background on ordered abelian groups, to [9] for the applications to von Neumann regular rings, and to [4], [6] and [14] for the applications to $\mathrm{C}^{*}$-algebras.

\section{BASIC RESUlts}

First, we recall some basics on abelian monoids. Let $M$ be an abelian monoid; we write $M^{*}$ to denote the set of non-zero elements of $M$. We say that $M$ is conical if, for all $x, y$ in $M$, $x+y=0$ only when $x=y=0$. A well-known example of conical, abelian monoid associated to a ring $R$ is the monoid $V(R)$ of equivalence classes of idempotents (see Section 4). We will use it in the last section of this paper. An element $x \in M$ is directly finite if for any $y \in M$, $x+y=x$ implies $y=0$. An element $x \in M$ is stably finite if $n x$ is directly finite for any $n \in \mathbb{N}$. We say that $M$ is stably finite if every element $x \in M$ is stably finite. A monoid $M$ is cancellative if, for all $x, y, z \in M$ such that $x+z=y+z$, we have $x=y$. If $x, y$ in $M$, we write $x \leq y$ if there exists $z \in M$ such that $x+z=y$; if $z \in M^{*}$, then we write $x<y$. Here $\leq$ is a translation-invariant pre-order on $M$. We say that $M$ is strictly unperforated if for 
any $n \in \mathbb{N}$ and for any $x, y \in M, n x<n y$ implies $x<y$; otherwise, $M$ is said to be strictly perforated. A non-zero element $u$ of $M$ is said to be an order-unit for $M$ if, for each $x \in M$, there exists a positive integer $n$ such that $x \leq n u$. If $u$ is an order-unit of $M$, then we call the pair $(M, u)$ a monoid with order-unit. Given $(M, u)$ and $(N, v)$ monoids with order-unit, a monoid morphism $f: M \rightarrow N$ is said to be normalized provided that $f(u)=v$. We say that $M$ is simple if $M$ is non-zero, conical, and every non-zero element is an order-unit. For other basic definitions and results on abelian monoids, see for example [19], [28] and [29].

Now, we recall some definitions (see, e.g. [19]) about monoids of intervals of conical monoids. Let $M$ be a conical monoid. A non-empty subset $X$ of $M$ is an interval in $M$ if $X$ is upwards directed and order-hereditary, i.e. if $x, y \in M$, such that $x \leq y$ and $y \in X$ then $x \in X$ (see [11], [12]). We denote by $\Lambda(M)$ the set of intervals in $M$. Note that $\Lambda(M)$ becomes an abelian monoid endowed with the operation defined by $X+Y=\{z \in M \mid z \leq x+y$ for some $x \in X, y \in Y\}$. An interval $X$ in $M$ is said to be generating if every element of $M$ is a sum of elements from $X$. If $X \in \Lambda(M)$, we say that $X$ is countably generated provided that $X$ has a countable cofinal subset (i.e. there is a sequence $\left\{x_{n}\right\}$ of elements in $X$ such that for any $x \in X$ there exists $n \in \mathbb{N}$ such that $x \leq x_{n}$ ). Notice that, since any interval is upwards directed, if $\left\{x_{n}\right\}$ is a countable cofinal subset generating an interval $X$, then we can choose a countable cofinal subset $\left\{y_{n}\right\}$ generating $X$ with the property that $y_{n} \leq y_{n+1}$ for all $n \geq 1$. We denote by $\Lambda_{\sigma}(M)$ the set of all countably generated intervals in $M$. If $D$ is a fixed interval in $\Lambda_{\sigma}(M)$, we denote by $\Lambda_{\sigma, D}(M)$ the submonoid of $\Lambda_{\sigma}(M)$ whose elements are intervals $X \in \Lambda_{\sigma}(M)$ such that $X \subseteq n D$ for some $n \in \mathbb{N}$, and we denote by $W_{\sigma}^{D}(M)$ the submonoid of $\Lambda_{\sigma, D}(M)$ whose elements are intervals $X \in \Lambda_{\sigma, D}(M)$ such that there exists $Y \in \Lambda_{\sigma, D}(M)$ with $X+Y=n D$ for some $n \in \mathbb{N}$.

Now, we proceed to state some basic results on intervals in a refinement monoid. They are analogous to results of [11], [13] or [19], but the original hypotheses are reduced to the minimum necessary.

Lemma 1.1. (c.f. [13, Lemma 3.8]) Let $M$ be a conical, cancellative monoid, and let $x \in M$. Then:

(1) $[0, x]=\{y \in M \mid y \leq x\}$ is an interval.

(2) If $X \in W_{\sigma}^{D}(M)$ and $x \in X$, then there exists an interval $Y \in W_{\sigma}^{D}(M)$ such that $[0, x]+Y=X$.

(3) If $D$ is a generating, countably generated interval, then $[0, x] \in W_{\sigma}^{D}(M)$.

Proof. It is obvious that $[0, x]$ is an interval. To prove (2), let $X \in W_{\sigma}^{D}(M)$ and assume that $x \in X$. Set $Y=\{y \in M \mid x+y \in X\}$. Clearly, $Y$ is non-empty and hereditary. Suppose that $y_{1}, y_{2} \in Y$. Then $x+y_{1}, x+y_{2} \in X$ whence there exists $z \in X$ such that $x+y_{1}, x+y_{2} \leq z$. Let $t_{1}, t_{2} \in M$ such that $x+y_{1}+t_{1}=z=x+y_{2}+t_{2}$. Since $M$ is cancellative, set $v=y_{1}+t_{1}=y_{2}+t_{2}$. Then $y_{1}, y_{2} \leq v$. Also $x+v=z \in X$, so that $v \in Y$, and thus $Y$ is an interval. Of course, $[0, x]+Y \subseteq X$ by definition. Now, take $z \in X$, and let $w \in X$ such that $x, z \leq w$. Thus there exist $a, b \in M$ with $x+a=w=z+b$. Then $a \in Y$, and $z \in[0, x]+[0, a] \subseteq[0, x]+Y$. It only remains to show that $Y$ is countably generated. But since $X$ is countably generated, and $M$ is cancellative, it is a trivial computation to show that so is $Y$. Hence (2) holds, and then we get (3) by taking $X=n D$ for a suitable $n \in \mathbb{N}$ so that $x \in n D$. 
If $M$ is a conical monoid, then we say that an interval $X$ in $M$ is soft if for each $x \in X$, there exist $y \in X$ and $n \in \mathbb{N}$ such that $(n+1) x \leq n y$.

Lemma 1.2. (c.f. [11, Lemma 7.4]) Let $M$ be a conical, stably finite, simple monoid, and let $D \subseteq M$ be an interval. Then $D$ is soft if and only if

$$
\text { For any } x \in D \text {, there exists } v \in M^{*} \text { such that } x+v \in D
$$

Proof. Assume that $(*)$ holds and let $x \in D$. Then we have $x+v=y \in D$. Since there exists $n \in \mathbb{N}$ with $x \leq n v$, we get $(n+1) x=n x+x \leq n x+n v=n(x+v)=n y$. Thus $D$ is soft. Now suppose that $D$ is soft, and $x$ be a non-zero element of $D$. By definition there exist $n \in \mathbb{N}$, $y \in D$ such that $(n+1) x \leq n y$. Since $D$ is an interval, there exists $z \in D$ with $x, y \leq z$. Thus $(n+1) x \leq n z$ and $x \leq z$. Let $t, t^{\prime} \in M$ such that $x+t=z$ and $(n+1) x+t^{\prime}=n z$. If $t=0$, then

$$
n x+\left(x+t^{\prime}\right)=(n+1) x+t^{\prime}=n z=n x,
$$

whence $x+t^{\prime}=0$, and thus $x=t^{\prime}=0$, which is impossible. Thus $t \neq 0$, and $x+t \in D$, as desired.

Note that we use the stably finite hypothesis only to prove that condition $(*)$ implies that $D$ is soft.

Lemma 1.3. (c.f. [11, Lemma 8.1]) Let $M$ be a conical, simple monoid, and let $D_{1}, D_{2} \subseteq M$ be intervals. If $D_{1}$ is soft, then so is $D_{1}+D_{2}$.

Proof. Let $x \in D_{1}+D_{2}$. Then there exist $x_{1} \in D_{1}, x_{2} \in D_{2}$ such that $x \leq x_{1}+x_{2}$. Let $\bar{x}=x_{1}+x_{2}$. Suppose that there exist $n \in \mathbb{N}, y \in D_{1}+D_{2}$ such that $(n+1) \bar{x} \leq n y$. Then $(n+1) x \leq n y$, so that, without loss of generality, we can assume that $x=x_{1}+x_{2}$ with $x_{1} \in D_{1}, x_{2} \in D_{2}$. Since $M$ is simple, the intervals $D_{1}, D_{2}$ are generating. Then the argument of [11, Lemma 8.1] applies, whence the desired result holds.

Lemma 1.4. (c.f. [19, Lemma 3.4]) Let $M$ be a conical, simple monoid. If $D$ is an interval, then it is either soft or of the form $[0, x]$ for some $x \in M$, but not both.

Proof. Suppose that there exists an element $x \in D$ such that for every $y \in D$ we have $x \nless y$. Take $y \in D$. Then there is $z \in D$ such that $x, y \leq z$. Since $x \nless z$, we have that $z=x$. Thus $D=[0, x]$.

Take $x \in D$ and find $y \in D$ and $t \in M^{*}$ such that $x+t=y \in D$. Since $M$ is simple, there exists $n \in \mathbb{N}$ such that $x \leq n t$. Thus, $(n+1) x=n x+x \leq n x+n t=n(x+t)=n y$. Hence $D$ is soft.

An element $a \in M^{*}$ is said to be an atom if for all $b \in M$, if $b \leq a$, then $b=a$ or $b=0$. A simple monoid $M$ is non-atomic if it has no atoms. We say that $M$ is a refinement monoid if, for all $a, b, c, d$ in $M$ such that $a+b=c+d$, there exist $w, x, y, z$ in $M$ such that $a=w+x$, $b=y+z, c=w+y$ and $d=x+z$. As we will see in Section 4 , if $R$ is a $C^{*}$-algebra of real rank zero, or a von Neumann regular ring, it is well-known that $V(R)$ is a refinement monoid (see, e.g. [9], [33], [1]). A special kind of soft intervals, very useful in the sequel, arises in case that $M$ is simple and non-atomic. 
Proposition 1.5. Let $M$ be a conical, cancellative, non-atomic, simple, refinement monoid, and let $x \in M^{*}$. Then the set

$$
[0, x)=\{y \in M \mid y<x\}
$$

is a soft interval. Furthermore, if $X$ is a countably generated soft interval and $x \in X$, then there exists a countably generated soft interval $Y$ such that $[0, x)+Y=X$. In particular, $[0, x) \in W_{\sigma}^{D}(M)$ whenever $D$ is soft and $M$ is countable.

Proof. The set $[0, x)$ is non-empty by construction. Clearly, $[0, x)$ is hereditary by definition. Now, let $y_{1}, y_{2} \in[0, x)$. By [10, Proposition 14.6], there exists $z \in M^{*}$ such that $y_{1}, y_{2}<z<x$. Hence $z \in[0, x)$, whence $[0, x)$ is upwards directed. Thus, $[0, x)$ is an interval. Moreover, by Lemma $1.2,[0, x)$ is soft.

Let $X$ be a countably generated soft interval with $x \in X$, and let $\left\{x_{n}\right\}_{n \geq 1}$ be a countable cofinal subset for $X$. By Lemma 1.4, we can assume that $x_{n}<x_{n+1}$ for all $n \geq 1$. Since $x \in X$, there exists $m \in \mathbb{N}$ such that $x \leq x_{m}$. Re-indexing if necessary we can assume without loss of generality that $m=1$. Let $y_{n} \in M$ be such that $x+y_{n}=x_{n}$ for any $n \geq 1$. Since $M$ is cancellative, the set $\left\{y_{n}\right\}_{n \geq 1}$ is an ascending chain. Let $Y=\bigcup_{n \geq 1}\left[0, y_{n}\right]$ be the interval generated by $\left\{y_{n}\right\}$. Notice that $Y$ is soft because of Lemma 1.2. By construction, we have $[0, x)+Y \subseteq X$. Conversely, let $a \in X$. Since $X$ is soft, there exists $b \in X$ such that $a, x<b$. As $b \in X$, there is $n \in \mathbb{N}$ so that $b<x_{n}$, and notice that $x_{n}=x+y_{n}$. Since $M$ is simple, cancellative and non-atomic, [10, Proposition 14.6] guarantees that there exist non-zero elements $b_{1}, b_{2} \in M$ such that $b=b_{1}+b_{2}$ with $b_{1}<x$ and $b_{2}<y_{n}$. Hence, $b \in[0, x)+\left[0, y_{n}\right] \subset[0, x)+Y$, and then also $a \in[0, x)+Y$. Thus $[0, x)+Y=X$.

Now assume that $M$ is countable. Since $M$ is simple, every non-zero interval is generating and countably generated. Thus, for any non-zero element $x \in M$ there is $n \in \mathbb{N}$ so that $x \in n D$. Since $D$ is soft, so is $n D$ by Lemma 1.3. Then $[0, x) \in W_{\sigma}^{D}(M)$ by the first part of the proof.

Remark 1.6. Under the hypotheses of Proposition 1.5, for any $x \in M^{*}$ we have $[0, x]=$ $[0, x) \cup\{x\}$. Thus, $[0, x)$ is the biggest soft interval contained in $[0, x]$. Also notice that, under the same hypotheses, we have that $[0, x)+[0, y)=[0, x+y)$ for any $x, y \in M$.

\section{Some SPECIAL SOFT INTERVALS}

We will use some techniques for representing intervals as functions on a compact space in order to get, in case of strictly perforated monoids, some special behaviors on soft intervals. For this, we recall some definitions. Given an abelian monoid $M$ and $x, y \in M$, we write $x \sim y$ if there exists $z \in M$ such that $x+z=y+z$. This is an equivalence relation on $M$, and we write $[x]$ for the equivalence class of an element $x \in M$. We define $G(M)=\{[x]-[y] \mid x, y \in M\}$, where $[a]-[b]=[c]-[d]$ if and only if $[a+d]=[c+b]$. Endowed with the operation $[x]+[y]=[x+y]$, the set $G(M)$ becomes a group, called the Grothendieck group of $M$. The submonoid $G(M)^{+}=\{[x] \mid x \in M\}$ is called the positive cone of $G(M)$, and induces an order on $G(M)$ as follows: given $x, y \in G(M)$, we say that $x \leq y$ if there exists $z \in G(M)^{+}$ such that $x+z=y$ (see [10]). If $M$ is cancellative then $G(M)^{+}=M$. Given a monoid with order-unit $(M, u)$, we denote $S_{u}$ the compact convex space of states (i.e. the set of monoid morphisms from $M$ to $\mathbb{R}^{+}$that send $u$ to 1$)$. Clearly, $S_{u}=S(G(M), u)$, the set of states 
on $G(M)$ (see [10]). We denote by $\operatorname{Aff}\left(S_{u}\right)^{+}$the monoid of positive, affine and continuous functions from $S_{u}$ to $\mathbb{R}^{+}$, endowed with the supremum norm, denoted $\|f\|$, and the natural pointwise ordering of functions. Given $f, g \in \operatorname{Aff}\left(S_{u}\right)^{+}$, we write $f \ll g$ if $f(s)<g(s)$ for all $s \in S_{u}$. We denote by $\phi_{u}: M \longrightarrow \operatorname{Aff}\left(S_{u}\right)^{+}$the natural evaluation map. Also, we denote by $\operatorname{LAff}_{\sigma}\left(S_{u}\right)^{++}$the semigroup of strictly positive, affine, lower semicontinuous functions from $S_{u}$ to $\mathbb{R}^{+}$that are pointwise suprema of increasing sequences of functions in $\operatorname{Aff}\left(S_{u}\right)^{+}$. Given an interval $X$ in $M$, we define $\rho(X)=\sup _{x \in X} \phi_{u}(x)$, where sup denotes the pointwise supremum.

Proposition 2.1. (c.f. [19, Lemma 3.8]) Let $M$ be a conical, cancellative, non-atomic, simple, refinement monoid, and let $u \in M^{*}$. Then:

(1) If $X \subseteq M$ is a non-zero interval, then $\rho(X) \in \operatorname{LAff}\left(\mathrm{S}_{\mathrm{u}}\right)^{++}$and it is bounded away from zero.

(2) $\rho(X)+\rho(Y)=\rho(X+Y)$ for any intervals $X, Y$ on $M$.

(3) If $f \in \operatorname{LAff}\left(\mathrm{S}_{\mathrm{u}}\right)^{++}$, then $\rho^{\prime}(f)=\left\{x \in M \mid \phi_{u}(x) \ll f\right\}$ is a soft interval in $M$, and $\rho \rho^{\prime}(f)=f$.

Proof. (1) and (2) are proved in the same way as [19, Lemma $3.8(1),(2)]$.

(3) Obviously the set $\rho^{\prime}(f)$ is non-empty and hereditary. Let $x, y \in \rho^{\prime}(f)$, and set $g=$ $\sup \left\{\phi_{u}(x), \phi_{u}(y)\right\}$. Clearly $g$ is an upper semicontinuous convex function, and $g \ll f$. By [10, Theorem 11.12] and [16, Theorem 3.5], there exists $z^{\prime} \in M$ such that $g \ll \phi_{u}\left(z^{\prime}\right) \ll f$. Since $\phi_{u}(x), \phi_{u}(y) \ll \phi_{u}\left(z^{\prime}\right)$, by $\left[10\right.$, Theorem 4.12] there exists $n \in \mathbb{N}$ such that $n x, n y<n z^{\prime}$. By compactness of $S_{u}$ we can choose $\varepsilon>0$ such that $\phi_{u}\left(z^{\prime}\right)+\varepsilon \ll f$. By [16, Corollary 2.6], there exists $d_{\varepsilon} \in M$ with $\left\|\phi_{u}\left(d_{\varepsilon}\right)\right\|<\varepsilon$, such that $x, y<z^{\prime}+d_{\varepsilon}$. Define $z=z^{\prime}+d_{\varepsilon}$. Then $z \in \rho^{\prime}(f)$, whence it is upwards directed, and so is an interval. Clearly is soft because of Lemma 1.2. The rest of the proof follows the argument of [19, Lemma $3.8(3)]$.

We denote $X^{\prime}=\rho^{\prime} \rho(X)$ for any interval $X$. Notice that, under the hypotheses of Proposition 2.1, $X^{\prime}=\rho^{\prime} \rho\left(X^{\prime}\right)$, because $\rho^{\prime} \rho\left(X^{\prime}\right)=\rho^{\prime} \rho \rho^{\prime} \rho(X)=\rho^{\prime} \rho(X)=X^{\prime}$. By [19, Lemma 3.8], for any soft interval $X$ in $M$ we have $\rho^{\prime} \rho(X)=X$, whenever $M$ is strictly unperforated. As we will see, when strict unperforation fails, we can construct soft intervals $X$ such that $X \neq \rho^{\prime} \rho(X)$. This is the key point of our arguments.

Lemma 2.2. Let $M$ be a conical, cancellative, non-atomic, simple, refinement monoid, and let $u \in M^{*}$. Let $X, Y \subseteq M$ be intervals. Then,

$$
\rho^{\prime}(\rho(X)+\rho(Y))=\rho^{\prime} \rho(X)+\rho^{\prime} \rho(Y)
$$

Proof. Let $f=\rho(X), g=\rho(Y)$, and let $x \in \rho^{\prime}(f+g)$. Then, $\phi_{u}(x) \ll f+g$, so that there exist $x_{0} \in X$ and $y_{0} \in Y$ with $\phi_{u}(x) \ll \phi_{u}\left(x_{0}\right)+\phi_{u}\left(y_{0}\right)$. By [10, Theorem 4.12], there exists $m \in \mathbb{N}$ with $m x<m x_{0}+m y_{0}$. Let $\varepsilon>0$ such that $\varepsilon<\min \left\{\left\|f-\phi_{u}\left(x_{0}\right)\right\|,\left\|g-\phi_{u}\left(y_{0}\right)\right\|\right\}$. By [16, Corollary 2.6] there exists $t \in M$ with $\left\|\phi_{u}(t)\right\|<\varepsilon$ and $x<\left(x_{0}+y_{0}\right)+t$. If $t=0$, then $x<x_{0}+y_{0} \in \rho^{\prime} \rho(X)+\rho^{\prime} \rho(Y)$. If $t \neq 0$, then since $M$ is non-atomic, there exist $t_{\varepsilon}, t_{\varepsilon}^{\prime} \in M$ such that $t_{\varepsilon}+t_{\varepsilon}^{\prime}=t$. Thus $\left\|\phi_{u}\left(t_{\varepsilon}\right)\right\|,\left\|\phi_{u}\left(t_{\varepsilon}^{\prime}\right)\right\|<\varepsilon$, and $x<\left(x_{0}+t_{\varepsilon}\right)+\left(y_{0}+t_{\varepsilon}^{\prime}\right)$. Moreover, $\phi_{u}\left(x_{0}+t_{\varepsilon}\right) \ll f$ and $\phi_{u}\left(x_{0}+t_{\varepsilon}^{\prime}\right) \ll g$. Hence $x_{0}+t_{\epsilon} \in \rho^{\prime} \rho(X)$ and $y_{0}+t_{\epsilon}^{\prime} \in \rho^{\prime} \rho(Y)$, so that $\rho^{\prime}(\rho(X)+\rho(Y)) \subseteq \rho^{\prime} \rho(X)+\rho^{\prime} \rho(Y)$.

Conversely, let $x \in \rho^{\prime}(f)+\rho^{\prime}(g)$. Then $x=y+z$, with $y \in \rho^{\prime}(f), z \in \rho^{\prime}(g)$, i.e., $\phi_{u}(y) \ll$ $f, \phi_{u}(z) \ll g$. Thus, $\phi_{u}(x)=\phi_{u}(y+z) \ll f+g$, whence $x \in \rho^{\prime}(f+g)=\rho^{\prime}(\rho(X)+\rho(Y))$, as required. 
Proposition 2.3. Let $M$ be a conical, cancellative, non-atomic, simple, refinement monoid, and let $u \in M^{*}$. If $X, Y$ are soft intervals, then

$$
X+Y^{\prime}=(X+Y)^{\prime}
$$

Proof. By Lemma 2.2 and Proposition 2.1,

$$
X+Y^{\prime} \subseteq X^{\prime}+Y^{\prime}=(X+Y)^{\prime} .
$$

Conversely, let $z \in(X+Y)^{\prime}$. Then there exist $x \in X^{\prime}$ and $y \in Y^{\prime}$ such that $\phi_{u}(z) \ll \phi_{u}(x)+$ $\phi_{u}(y)$. By definition of $X^{\prime}, \phi_{u}(x) \ll \rho(X)$. Analogously $\phi_{u}(y) \ll \rho(Y)$. By compactness of $S_{u}$, there exists $\varepsilon>0$ such that

$$
\varepsilon<\frac{1}{2} \min \left\{\left\|\rho(X)-\phi_{u}(x)\right\|,\left\|\rho(Y)-\phi_{u}(y)\right\|\right\} .
$$

By [10, Theorem 4.12] and [16, Corollary 2.6], there exist $d, d^{\prime} \in M$ such that

$$
\left\|\phi_{u}(d)\right\|,\left\|\phi_{u}\left(d^{\prime}\right)\right\|<\varepsilon
$$

and $z<(x+d)+\left(y+d^{\prime}\right)$. Notice that $x+d \in X^{\prime}$ and $y+d^{\prime} \in Y^{\prime}$. By [10, Proposition 14.6], we get decompositions $z=z_{1}+z_{2}$ with $z_{1}<(x+d), z_{2}<\left(y+d^{\prime}\right)$. By the argument in the proof of [11, Proposition 7.7], there exists $t \in X$ such that $\phi_{u}\left(z_{1}\right) \ll \phi_{u}(t)$. Let $\varepsilon^{\prime}>0$ be such that $\phi_{u}\left(z_{2}\right)+\varepsilon^{\prime} \ll \rho(Y)$. By [16, Theorem 4.12], there exist decompositions $z_{1}=z_{3}+z_{4}, t=z_{5}+z_{4}$ with $\left\|\phi_{u}\left(z_{3}\right)\right\|<\varepsilon^{\prime}$. Suppose $z_{5}=0$. Then $t=z_{4}$, and hence $z_{1}=z_{3}+z_{4}=z_{3}+t$. But then $\phi_{u}(t) \leq \phi_{u}\left(z_{1}\right) \ll \phi_{u}(t)$, which is impossible. Thus, $z=z_{2}+z_{3}+z_{4}$, with $z_{4}<t \in X$, and

$$
\phi_{u}\left(z_{2}+z_{3}\right)=\phi_{u}\left(z_{2}\right)+\phi_{u}\left(z_{3}\right) \ll \phi_{u}\left(z_{2}\right)+\varepsilon^{\prime} \ll \rho(Y) .
$$

Hence $z_{4} \in X$ and $z_{2}+z_{3} \in Y^{\prime}$, as required.

As a consequence we have

Corollary 2.4. Let $M$ be a conical, cancellative, non-atomic, simple, refinement monoid, and let $u \in M^{*}$. If $X$ is a soft interval, then

$$
X+X^{\prime}=2 X^{\prime} .
$$

Let $M$ be a monoid. In order to study the properties of a separative positively ordered monoid, Wehrung [29] defined the equivalence relation $\asymp$ for $a, b \in M: a \asymp b$ if and only if there exist $m, n \in \mathbb{N}$ such that $a \leq m b, b \leq n a$.

Lemma 2.5. Let $M$ be a conical, cancellative, non-atomic, simple, refinement monoid, and let $u \in M^{*}$. If $x, y \in M^{*}$, then:

(1) $[0, x) \asymp[0, y)$.

(2) $\rho^{\prime} \rho([0, x)) \asymp \rho^{\prime} \rho([0, y))$.

(3) $\rho^{\prime} \rho([0, x)) \asymp[0, x)$ if and only if there exists $n_{0} \in \mathbb{N}$ such that for any $n \geq n_{0}$, $\rho^{\prime} \rho([0, n x))=[0, n x)$.

Proof. (1) Since $M$ is simple, there exist $m \in \mathbb{N}, z, t \in M$ such that $x+t=m y, y+z=m x$. Hence $[0, x)+[0, t)=m[0, y)$ and $[0, y)+[0, z)=m[0, x)$, as desired.

(2) Using (1), Lemma 2.2, Proposition 2.1 (2) and Proposition 2.3, we get $\rho^{\prime} \rho([0, x))+$ $[0, t)=m \rho^{\prime} \rho([0, y))$ and $\rho^{\prime} \rho([0, y))+[0, z)=m \rho^{\prime} \rho([0, x))$.

(3) Suppose that $\rho^{\prime} \rho([0, x)) \asymp[0, x)$. By Proposition $1.5,[0, x)$ is a soft interval. Then by Corollary 2.4, we have $[0, x)+\rho^{\prime} \rho([0, x))=2 \rho^{\prime} \rho([0, x))$. By [5, Theorem 2.1.9] there exists 
$n_{0} \in \mathbb{N}$ such that, for any $n \geq n_{0}$, we have $n \rho^{\prime} \rho([0, x))=n[0, x)$. By Lemma 2.2, Proposition 2.1 (2) and Remark 1.6, we have $n \rho^{\prime} \rho([0, x))=\rho^{\prime}(n \rho([0, x)))=\rho^{\prime} \rho(n[0, x))=\rho^{\prime} \rho([0, n x))$. Then, $\rho^{\prime} \rho([0, n x))=[0, n x)$. The converse is obvious.

The next result allows us to construct the pathological soft intervals we need in the sequel.

Lemma 2.6. Let $M$ be a conical, cancellative, non-atomic, strictly perforated, simple, refinement monoid. Then there exist $n \in \mathbb{N}$ and $x \in G(M)$ such that $n x$ and $(n+1) x \in G(M)^{+}$ but $n x \notin[0,(n+1) x)$.

Proof. Since $M$ is strictly perforated, there exists $x \in G(M) \backslash\{0\}$ such that $x \notin G(M)^{+}$ and $n x \in G(M)^{+}$for some $n \in \mathbb{N}$. We may assume that $(n+1) x \notin G(M)^{+}$, as otherwise $x \in G(M)^{+}$. As $n x$ is an order-unit, there exists $k \in \mathbb{N}$ with $(n+1) x \leq k n x$. Then, $[(k-1) n-1] x \in G(M)^{+}$. Since $[(k-1) n-1]$ is 1 minus a multiple of $n$, we have g.c.d.([(k$1) n-1], n)=1$. Hence, there exist $p, q \in \mathbb{N}$ such that $p n-q[(k-1) n-1]=1$, whence $p n=1+q[(k-1) n-1]$. Set $m=q[(k-1) n-1]$. Then, $m x=q[(k-1) n-1] x \in G(M)^{+}$and $(m+1) x=(1+q[(k-1) n-1]) x=p n x \in G(M)^{+}$. On the other hand, $m x \notin[0,(m+1) x)$, as otherwise, we get $x \in G(M)^{+}$, which is impossible.

Proposition 2.7. Let $M$ be a conical, cancellative, non-atomic, strictly perforated, simple, refinement monoid, and let $u \in M^{*}$. Then, for any $x, y \in M^{*},[0, x) \nprec \rho^{\prime} \rho([0, y))$.

Proof. By conditions (1) and (2) in Lemma 2.5, it is enough to find an element $x \in M^{*}$ for which $[0, x) \nprec \rho^{\prime} \rho([0, x))$. By Lemma 2.6, there exist $n \in \mathbb{N}$ and $y \in G(M)$, such that $y \notin G(M)^{+}$but $n y,(n+1) y \in G(M)^{+}$. If $N=n(n+1)-2 n-1$, then for any $k \in \mathbb{N}$ we have $(N+k) y \in G(M)^{+}$(see, e.g. [21, Lemma 2.3]). Set $x=(n+1) y$.

If $[0, x) \asymp \rho^{\prime} \rho([0, x))$, by Lemma 2.5 there exists $m_{0} \in \mathbb{N}$ such that $[0, m x)=\rho^{\prime} \rho([0, m x))$ for all $m \geq m_{0}$. Take $m \geq m_{0}$ so that $(m+1)(n+1) \geq N+2$. Thus, $\rho^{\prime} \rho([0,(m+1) x))=$ $[0,(m+1) x)$. Set $z=[(m+1)(n+1)-1] y$, and notice that $z \in G(M)^{+}$. Since $y \notin G(M)^{+}$, we have $z \notin[0,(m+1) x)$. This contradicts $[0, m x)=\rho^{\prime} \rho([0, m x))$ for all $m \geq m_{0}$. Hence, $[0, x) \nprec \rho^{\prime} \rho([0, x))$, as desired.

Corollary 2.8. Let $M$ be a conical, cancellative, non-atomic, strictly perforated, simple, refinement monoid, and let $u \in M^{*}$. Then, $[0, x) \neq \rho^{\prime} \rho([0, x))$ for any $x \in M^{*}$.

\section{The Functional REPRESENTAtion}

We recall the key result of Perera's work on the structure of the monoid of intervals of a non-atomic, strictly unperforated, simple, refinement monoid.

Theorem 3.1. ([19, Theorem 3.9]) Let $M$ be a conical, simple, refinement monoid, let $u \in$ $M^{*}$, let $D$ be a countably generated soft interval in $M$, and let $d=\rho(D)$. Define

$$
\begin{aligned}
\varphi: W_{\sigma}^{D}(M) & \longrightarrow M \sqcup W_{\sigma}^{d}\left(S_{u}\right) \\
X & \longmapsto \rho(X) \text { if } X \text { is a soft interval } \\
{[0, x] } & \longmapsto x \text { if } x \in M .
\end{aligned}
$$

Then $\varphi$ is a normalized monoid morphism. Moreover, if $M$ is non-atomic, strictly unperforated and cancellative, then $\varphi$ is an isomorphism. 
We say that a soft interval $X$ in $M$ is functionally complete whenever $\rho^{\prime} \rho(X)=X$. Injectivity and surjectivity of the map $\varphi$ strongly depend on the fact that, when $M$ is strictly unperforated, any soft interval $X$ in $M$ is functionally complete ([19, Lemma $3.8(4)])$. It is always true that $X \subseteq \rho^{\prime} \rho(X)$. Nevertheless, as we have seen in Corollary 2.8, this equality may fail when $M$ is strictly perforated.

Now, we will show that under some mild hypotheses, this map is a monomorphism if and only if $M$ is strictly unperforated, and that it is always an epimorphism. Recall that, if $M$ is a (semigroup) monoid, we say that $M$ is separative if it satisfies the weak cancellation condition that, for all $a, b$ in $M, a+a=a+b=b+b$ only if $a=b$. Similarly, we say that $M$ is strongly separative if it satisfies the weak cancellation condition that, for all $a, b$ in $M$, $a+a=a+b$ only if $a=b$.

We define $\mathcal{E}_{\text {fin }}=\left\{f \in W_{\sigma}^{D}(M) \mid f_{\partial_{e} S_{u}}\right.$ is finite $\}$, where $\partial_{e} S_{u}$ denotes the extreme boundary of $S_{u}$ (see [10]).

Theorem 3.2. Let $M$ be a countable, conical, cancellative, non-atomic, simple, refinement monoid, and let $u \in M^{*}$. Then the following are equivalent:

(1) $M$ is strictly unperforated.

(2) Every soft interval $X \subseteq M$ is functionally complete.

(3) The morphism $\varphi$ of Theorem 3.1 is injective for any soft interval $D$ in $M$.

(4) For any soft interval $D$ in $M$, the subsemigroup $E=\varphi^{-1}\left(\mathcal{E}_{\text {fin }}\right)$ of $W_{\sigma}^{D}(M)$ is strongly separative.

(5) For any $x \in M^{*},[0, x)$ is functionally complete.

Proof. (1) $\Rightarrow(2)$ This is [19, Lemma 3.8 (4)].

$(2) \Rightarrow(1)$ Suppose that $M$ is strictly perforated. By Corollary $2.8,[0, x) \varsubsetneqq \rho^{\prime} \rho([0, x))$ for any $x \in M^{*}$.

$(1) \Rightarrow(3)$ This is Theorem 3.1 .

$(3) \Rightarrow(1)$ Suppose that $M$ is strictly perforated. As in the proof of $(2) \Rightarrow(1)$, there exist $y, z \in M$ such that $z \notin[0, y)$ but $\phi_{u}(z) \ll \phi_{u}(y)$. Let $X=[0, y), X^{\prime}=\rho^{\prime} \rho(X)$. Fix a soft interval $D$ such that $\rho^{\prime} \rho(D)=D$ (take for example an interval of the form $\rho^{\prime} \rho(I)$, for any interval $I$ in $M)$. By Proposition 1.5, $X \in W_{\sigma}^{D}(M)$, and thus there exist $Y \in W_{\sigma}^{D}(M)$ and $n \in \mathbb{N}$ such that $X+Y=n D$. By Lemma 2.2 and Proposition 2.1,

$$
\rho^{\prime} \rho(X)+\rho^{\prime} \rho(Y)=n \rho^{\prime} \rho(D)=n D .
$$
$(3)$,

Hence $X^{\prime} \in W_{\sigma}^{D}(M)$. Since $z \notin X$, but $z \in X^{\prime}$, we have $X \varsubsetneqq X^{\prime}$ and by Proposition 2.1

$$
\varphi\left(X^{\prime}\right)=\rho\left(X^{\prime}\right)=\rho\left(\rho^{\prime} \rho(X)\right)=\rho \rho^{\prime}(\rho(X))=\rho(X)=\varphi(X),
$$

whence $\varphi$ is not injective.

$(1) \Rightarrow(4)$ It is Theorem 3.1 .

$(4) \Rightarrow(1)$ Suppose that $M$ is strictly perforated. As in the proof of $(3) \Rightarrow(1)$, there exists $x \in M^{*}$ such that, if $X=[0, x)$, then $X \neq X^{\prime}$. On the other hand, $X+X^{\prime}=2 X^{\prime}$ by Corollary 2.4. Moreover $X \in E$ by definition, whence the result holds.

$(2) \Rightarrow(5)$ It is obvious, since $[0, x)$ is a soft interval by Proposition 2.1 .

$(5) \Rightarrow(1)$ It is the same proof as $(2) \Rightarrow(1)$. 
Notice that we only need the hypothesis " $M$ is a countable monoid" to show $(3) \Rightarrow(1)$ and $(4) \Rightarrow(1)$. Because of its own interest, we will state $(4) \Rightarrow(1)$ in Theorem 3.2 as follows:

Corollary 3.3. Let $M$ be a countable, conical, cancellative, non-atomic, strictly perforated, simple, refinement monoid, and let $D$ be a non-zero functionally complete soft interval in $M$. Then, the subsemigroup $E=\varphi^{-1}\left(\mathcal{E}_{\text {fin }}\right)$ of $W_{\sigma}^{D}(M)$ fails to be strongly separative.

\section{Remark 3.4.}

(1) Perera [19] showed that, whenever we consider a strictly unperforated simple Riesz group, its monoid of intervals satisfy the separativity property. On the other hand, Wehrung [32, Example 3.14] constructed a torsion free simple Riesz group $G$, whose positive cone is strictly perforated, containing an interval $D \neq G^{+}$such that $2 D=G^{+}$. Thus, the monoid of intervals $W_{\sigma}^{D}\left(G^{+}\right)$fails separativity. Nevertheless, we cannot guarantee that given a strictly perforated, simple, refinement monoid $M$, its monoid of intervals $W_{\sigma}^{D}(M)$ will always be non-separative. For example, Pardo [15, Section 3] gave examples of torsion free simple Riesz groups $G$, whose positive cones are strictly perforated, and with the property that any interval $D$ such that $\rho(D)=\rho\left(G^{+}\right)$implies $D=G^{+}$. Then, in order to check if such examples fail separativity, we need to study carefully the behavior of intervals $D$ such that $n D \neq G^{+}$for every $n \in \mathbb{N}$. Thus, it remains as an open question

Given any countable, simple refinement monoid $M$ that fails to be strictly perforated, is $W_{\sigma}^{D}(M)$ always non-separative?

(2) Notice that, in the proof of Theorem $3.2(3) \Rightarrow(1)$, a key point is to choose a functionally complete interval $D$ to show the failure of injectivity for the map $\varphi$. Even if we take a non functionally complete interval $D$ as order-unit for $W_{\sigma}^{D}(M)$, it is possible to show the failure of injectivity for the map $\varphi$ in some special cases. Wehrung's example [32, Example 3.14] fits one possibility. Another possibility is the following: suppose that there exists a simple Riesz group $\left(G, G^{+}\right)$containing an interval $D \subseteq G^{+}$such that $\rho(D)=\rho\left(G^{+}\right)$, but for every $n \in \mathbb{N}, n D \neq G^{+}$(in particular $G$ is strictly perforated). Then, $W_{\sigma}^{D}\left(G^{+}\right)$is stably finite, so that $n D \neq m D$ whenever $n \neq m$, but $\varphi(n D) \equiv \infty$. In terms of the K-Theory of multiplier algebras (see Section 4), the existence of such a group implies that it could be possible to construct a $\sigma$-unital, non-unital, simple $\mathrm{C}^{*}$-algebra with real rank zero and stable rank one $A$, such that its multiplier algebra $\mathcal{M}(A)$ contains a non-zero projection $P$ with $\mathcal{M}(P A P)$ stably finite, but with identically infinite scale ([19]). Thus, according to [20, Proposition 3.6] (also see [17, Theorem 2.10]), PAP is not an stable algebra. The existence of such an example will fix the exact limits of application of [17, Proposition 2.11].

Theorem 3.5. Let $M$ be a conical, cancellative, non-atomic, simple, refinement monoid, let $u \in M^{*}$, and let $D$ be a non-zero, functionally complete, countably generated, soft interval of $M$. Then the normalized morphism of Theorem 3.1 is onto.

Proof. If $x \in M$, then let $X=[0, x] \in W_{\sigma}^{D}(M)$, so that $\varphi(X)=x$. Now, let $f \in W_{\sigma}^{d}\left(S_{u}\right)$. Then, there exists $h \in W_{\sigma}^{d}\left(S_{u}\right)$ such that $f+h=n d$, where $d=\rho(D), n \in \mathbb{N}$. We have $f=\sup g_{n}$, where $\left\{g_{n}\right\}$ is an ascending sequence of functions in $\operatorname{Aff}\left(S_{u}\right)^{++}$. By [16, Theorem $3.5]$, there is a $m \in \mathbb{N}$ such that, for $n \geq m$, there exists $x_{n} \in M$ with $0 \ll g_{n}-\frac{1}{2^{n}} \ll$ 
$\phi_{u}\left(x_{n}\right) \ll g_{n+1}-\frac{1}{2^{n+1}}$. Then,

$$
0 \ll g_{n}-\frac{1}{2^{n}} \ll \phi_{u}\left(x_{n}\right) \ll g_{n+1}-\frac{1}{2^{n+1}} \ll \phi_{u}\left(x_{n+1}\right) \ll g_{n+2}-\frac{1}{2^{n+2}} \ll \cdots
$$

By compactness of $S_{u}$ we get, for each $n \in \mathbb{N}$, an $\varepsilon_{n}>0$ such that $\phi_{u}\left(x_{n+1}\right)+\varepsilon_{n} \ll g_{n+2}-\frac{1}{2^{n+2}}$ (see, e.g. [11, Proposition 7.7]). Then using [16, Theorem 4.12], we get $b \in M$ such that $x_{n}<x_{n+1}+b$ and $\left\|\phi_{u}(b)\right\|<\varepsilon_{n}$. Thus,

$$
0 \ll g_{n}-\frac{1}{2^{n}} \ll \phi_{u}\left(x_{n}\right) \ll g_{n+1}-\frac{1}{2^{n+1}} \ll \phi_{u}\left(x_{n+1}+b\right) \ll g_{n+2}-\frac{1}{2^{n+2}} \ll \cdots
$$

Define $y_{n}=x_{n}, y_{n+1}=x_{n+1}+b$. Then, for $y_{n+1}$ and $x_{n+2}$ we have

$$
\cdots \ll g_{n+1}-\frac{1}{2^{n+1}} \ll \phi_{u}\left(y_{n+1}\right) \ll g_{n+2}-\frac{1}{2^{n+2}} \ll \phi_{u}\left(x_{n+2}\right) \ll g_{n+3}-\frac{1}{2^{n+3}} \ll \cdots
$$

By recurrence on this procedure we get an ascending sequence $\left\{y_{n}\right\}_{n \in \mathbb{N}}$ with $f=\sup \phi_{u}\left(y_{n}\right)$. Let $X$ be the interval generated by $\left\{y_{n}\right\}$ (in particular, it is countably generated). Thus, $X=\bigcup_{n \in \mathbb{N}}\left[0, y_{n}\right]$, so that $\rho(X)=f$. As $\phi_{u}\left(y_{n}\right) \ll f$ for all $n$, we have $X \subseteq \rho^{\prime}(f)$, and $X$ is soft by Lemma 1.4.

It only remains to show that $X \in W_{\sigma}^{D}(M)$. Since $f+h=n d$, we apply the same argument to $h$, and we construct a countably generated, soft interval $Z$ such that $\rho(Z)=h$, so that $Z \subseteq \rho^{\prime}(h)$. By Corollary 2.4,

$$
\begin{aligned}
X+\rho^{\prime} \rho(X) & =2 \rho^{\prime} \rho(X) \\
Z+\rho^{\prime} \rho(Z) & =2 \rho^{\prime} \rho(Z)
\end{aligned}
$$

Hence,

$$
X+\rho^{\prime} \rho(X)+Z+\rho^{\prime} \rho(Z)=2\left(\rho^{\prime} \rho(X)+\rho^{\prime} \rho(Z)\right),
$$

whence by Lemma 2.2 ,

$$
X+Z+\rho^{\prime}(\rho(X)+\rho(Z))=2 \rho^{\prime}(\rho(X)+\rho(Z)),
$$

that is,

Since $f+h=n d$,

$$
X+Z+\rho^{\prime}(f+h)=2 \rho^{\prime}(f+h) .
$$

As $d=\rho(D)$,

$$
X+Z+\rho^{\prime}(n d)=2 \rho^{\prime}(n d) .
$$

whence by Lemma 2.2,

$$
X+Z+\rho^{\prime}(n \rho(D))=2 \rho^{\prime}(n \rho(D)),
$$

Since $\rho^{\prime} \rho(D)=D$, we have

$$
X+Z+n \rho^{\prime} \rho(D)=2 n \rho^{\prime} \rho(D)
$$

$$
X+Z+n D=2 n D .
$$

Finally, as $Z$ and $D$ are countably generated, so is $Z+n D$, whence $X \in W_{\sigma}^{D}(M)$.

As an immediate consequence of Theorem 3.2 and Theorem 3.5, we get

Corollary 3.6. Let $M$ be a countable, conical, cancellative, non-atomic, strictly perforated, simple, refinement monoid, let $u \in M^{*}$, and let $D \subseteq M$ be a non-zero functionally complete soft interval. Then the map $\varphi$ of Theorem 3.1 is always onto, but never injective. 


\section{Remark 3.7.}

(1) Notice that for any monoid with order-unit $(M, u)$, we have that $\rho^{\prime} \rho(M)=M$, and $M$ is a countably generated interval, because $\{n u \mid n \in \mathbb{N}\}$ generates it as an interval. Then, for any monoid there is at least one interval satisfying the hypothesis of Corollary 3.6, whence Theorem 3.5 applies at least in this case.

(2) Recall that a subset $S$ of a monoid $M$ is called an order-ideal, or simply an ideal, if $S$ is a subset of $M$ containing 0 , closed under taking sums and summands within $M$; that is, $S$ is a submonoid such that, for all $x \in M$ and $e \in S$, if $x \leq e$ then $x \in S$. For any $a \in M$, the principal ideal generated by $a$ is $M(a)=\{x \in M \mid$ $x \leq n a$ for some positive integer $n\}$. We denote the set of ideals of $M$ by $L(M)$. If $M$ is a refinement monoid then, by [1, Lemma 2.1], $L(M)$ forms a lattice under sum and intersection. Perera used [19, Theorem 3.9] in order to describe this lattice in terms of the behavior of the extremal states on $S_{u}$. As a consequence of Corollary 2.8 , even in the case of principal ideals, for any $x \in M^{*}$ we have $W_{\sigma}^{D}(M)([0, x)) \subset$ $W_{\sigma}^{D}(M)\left(\rho^{\prime} \rho([0, x))\right)$. Thus, Perera's description of $L\left(W_{\sigma}^{D}(M)\right)$ is no longer valid. Nevertheless, in the case $\varphi$ being onto, if we are able to describe the set $\varphi^{-1}(f)$, it seems reasonable to recover the structure of this lattice.

Finally, even if injectivity fails, we still preserve a certain "good" behavior of divisibility for soft intervals, in a similar way to that of [13, Lemma 4.2(b)].

Proposition 3.8. Let $M$ be a conical, non-atomic, simple, refinement monoid. For any $n \in \mathbb{N}$ and any non-zero soft interval $X \in W_{\sigma}^{D}(M)$, there exists $Y \in W_{\sigma}^{D}(M)$ such that $X=n Y$.

Proof. The proof is an adaptation of that of [35, Theorem I (ii)], using [16, Theorem 5.2].

Let $X$ be the interval generated by the ascending sequence $\left\{x_{i}\right\}$, let $p_{i} \in X$ such that $x_{i-1}+p_{i}=x_{i}$ for any $i>1$, and set $p_{1}=x_{1}$. Thus, $\sum_{i=1}^{k} p_{i}=x_{k}$. Applying [16, Theorem $5.2]$ to $p_{1}$ and $p_{2}$, we get $r_{1}, s_{1} \in M$ with $p_{1}=n r_{1}+s_{1}$ and $(n-1) s_{1}<p_{2}$. Hence, there exists $z_{2} \in M$ such that $(n-1) s_{1}+z_{2}=p_{2}$. Since $r_{1}, s_{1} \leq p_{1}$ and $z_{2} \leq p_{2}$, we have $r_{1}, s_{1}, z_{2} \in X$.

Applying again [16, Theorem 5.2] to $z_{2}$ and $p_{3}$, we get $s_{2}, r_{2} \in M$ with $z_{2}=n r_{2}+s_{2}$ and $(n-1) s_{2}<p_{3}$. Hence, there exists $z_{3} \in M$ such that $(n-1) s_{2}+z_{3}=p_{3}$. Since $r_{2}, s_{2} \leq p_{2}$ and $z_{3} \leq p_{3}$, we have $r_{2}, s_{2}, z_{3} \in X$.

By recurrence on this argument we obtain

$$
z_{i}=n r_{i}+s_{i} \text { with }(n-1) s_{i}+z_{i+1}=p_{i+1}, i>1, z_{1}=p_{1} .
$$

Defining $y_{i}=\sum_{j=1}^{i}\left(r_{j}+s_{j-1}\right)$ for $i \geq 1$, and $s_{0}=0$, we get an ascending chain $\left\{y_{i}\right\}$. Set $Y$ the interval generated by $\left\{y_{i}\right\}$. Then,

$$
\begin{gathered}
n y_{i}=n \sum_{j=1}^{i}\left(r_{j}+s_{j-1}\right)=n s_{0}+n r_{i}+\sum_{j=1}^{i-1}\left(n r_{j}+s_{j}\right)+\sum_{j=1}^{i-1}(n-1) s_{j}= \\
n r_{i}+\sum_{j=1}^{i-1} z_{j}+\sum_{j=1}^{i-1}(n-1) s_{j}=n r_{i}+(n-1) s_{i-1}+\sum_{j=1}^{i-1}\left(z_{j}+(n-1) s_{j-1}\right)=
\end{gathered}
$$




$$
\sum_{j=1}^{i}\left(z_{j}+(n-1) s_{j-1}\right)=\sum_{j=1}^{i} p_{j}=x_{i}
$$

so that $n y_{i} \in X$. Also, for each $i \geq 1$,

$$
\begin{gathered}
x_{i}=\sum_{j=1}^{i} p_{j}=\sum_{j=1}^{i}\left(z_{j}+(n-1) s_{j-1}\right)=\sum_{j=1}^{i}\left(n r_{j}+s_{j}+(n-1) s_{j-1}\right)= \\
\sum_{j=1}^{i}\left((n-1) r_{j}+r_{j}+s_{j}+(n-1) s_{j-1}\right)=\sum_{j=1}^{i}\left((n-1) s_{j-1}+(n-1) r_{j}\right)+\sum_{j=1}^{i}\left(r_{j}+s_{j}\right)= \\
(n-1) \sum_{j=1}^{i}\left(s_{j-1}+r_{j}\right)+\sum_{j=1}^{i}\left(r_{j}+s_{j}\right) \leq(n-1) y_{i}+\sum_{j=1}^{i}\left(r_{j}+s_{j}\right)+r_{i+1}= \\
(n-1) y_{i}+\sum_{j=1}^{i+1}\left(r_{j}+s_{j-1}\right)=(n-1) y_{i}+y_{i+1} \leq n y_{i+1} \in n Y .
\end{gathered}
$$

Hence, $X=n Y$, as desired.

Notice that, in spite of [13, Lemma 4.2(b)], Proposition 3.8 does not guarantee the uniqueness of the interval $Y$.

\section{Applications to MUltiplier Rings}

In this section we apply the results we obtained in the previous sections to context of non-stable K-Theory of multiplier rings for non-unital von Neumann regular rings and $\mathrm{C}^{*}$ algebras. We start by recalling some definitions and results of [3], [13] and [19].

Given a ring $R$, we denote by $M_{\infty}(R)=\lim M_{n}(R)$, under the maps $M_{n}(R) \rightarrow M_{n+1}(R)$ defined by $x \mapsto \operatorname{diag}(x, 0)$. Notice that $M_{\infty}(\overrightarrow{R)}$ can also be described as the ring of countable infinite matrices over $R$ with only finitely many nonzero entries. Given $p, q \in M_{\infty}(R)$ idempotents, we say that $p$ and $q$ are equivalent, denoted $p \sim q$, if there exist elements $x, y \in M_{\infty}(R)$ such that $x y=p$ and $y x=q$. We also write $p \leq q$ provided that $p=p q=q p$, and we write $p \lesssim q$ if there exists an idempotent $r \in M_{\infty}(R)$ such that $p \sim r \leq q$. Given idempotents $p, q \in M_{\infty}(R)$, we define the direct sum of $p$ and $q$ as $p \oplus q=\left(\begin{array}{cc}p & 0 \\ 0 & q\end{array}\right)$. Also, for an idempotent $p \in M_{\infty}(R)$ and a positive integer $n$, we denote by $n \cdot p$ the direct sum of $\mathrm{n}$ copies of $p$. For a ring $R$, we denote by $V(R)$ the abelian monoid of equivalence classes of idempotents in $M_{\infty}(R)$ under the relation $\sim$ defined above, with the operation $[p]+[q]=[p \oplus q]$. We consider this monoid endowed with the algebraic pre-ordering, denoted by $\leq$, that corresponds to the ordering induced by the relation $\lesssim$. Given a ring $R$, it is easy to see that $V(R)$ is conical, and if $R$ is simple, then so is $V(R)$. Also, if $R$ is a separable $\mathrm{C}^{*}$-algebra or a countable ring, then $V(R)$ is a countable monoid. In the case of a $\mathrm{C}^{*}$-algebra $A$, we can also obtain a picture of $V(A)$ by considering the equivalence classes of projections (self-adjoint idempotents) in $M_{\infty}(A)$, under the same equivalence relation we introduced before (see, e.g. [4, Chapter 5]).

Given a ring $R$, we say that a double centralizer for $R$ is a pair $(\mathcal{L}, \mathcal{R})$ of additive maps $\mathcal{L}, \mathcal{R}: R \rightarrow R$ satisfying $\mathcal{R}(x) y=x \mathcal{L}(y)$ for all $x, y \in R$. Notice that for any element $a \in R$, the pair $\left(\mathcal{L}_{a}, \mathcal{R}_{a}\right)$, where the maps are left/right multiplication by a respectively, is 
a double centralizer. The set of double centralizers over $R$, endowed with the operations $\left(\mathcal{L}_{1}, \mathcal{R}_{1}\right)+\left(\mathcal{L}_{2}, \mathcal{R}_{2}\right)=\left(\mathcal{L}_{1}+\mathcal{L}_{2}, \mathcal{R}_{1}+\mathcal{R}_{2}\right)$ and $\left(\mathcal{L}_{1}, \mathcal{R}_{1}\right) \cdot\left(\mathcal{L}_{2}, \mathcal{R}_{2}\right)=\left(\mathcal{L}_{1} \cdot \mathcal{L}_{2}, \mathcal{R}_{2} \cdot \mathcal{R}_{1}\right)$, has structure of ring with unit (Id, Id), and it is called the ring of multipliers of $R$, denoted $\mathcal{M}(R)$. Notice that $R$ is an ideal of $\mathcal{M}(R)$ through the identification of $a \in R$ with $\left(\mathcal{L}_{a}, \mathcal{R}_{a}\right) \in \mathcal{M}(R)$; moreover, $\mathcal{M}(R)$ coincides with $R$ whenever $R$ is a unital ring. In the case of $A$ being a $\mathrm{C}^{*}$ algebra, it is well-known that $\mathcal{M}(A)$ is also a $\mathrm{C}^{*}$-algebra (see, e.g. [27]).

Let $R$ be a ring, and let $S$ be a unital ring containing $R$ as a two-sided ideal (for example, we can choose $S=\mathcal{M}(R)$ ). Then we say that $R$ has stable rank one (denoted $\operatorname{sr}(R)=1$ ) if, whenever $S a+S b=S$ with $a-1, b \in R$, there exists $t \in R$ such that $S(a+t b)=S$. This definition does not depend on the choice of $S$ [23]; moreover, if $\operatorname{sr}(R)=1$ and $e \in R$ is an idempotent, then $\operatorname{sr}(e R e)=1$ [24, Theorem 3.9]. Since for a unital ring $R$ it is well-known that $\operatorname{sr}(R)=1$ implies that $V(R)$ is a cancellative monoid (see [8]), we conclude by [24, Theorem 3.9] that if $R$ is a non-unital ring, then $V(R)$ is also a cancellative monoid.

A $C^{*}$-algebra $A$ has real rank zero provided that the set of invertible self-adjoint elements of $A$ is dense in the set of self-adjoint elements of $A$ (see [6]). According to [1], this is equivalent to the fact that the $\mathrm{C}^{*}$-algebra $A$ is an exchange ring in the sense of Warfield [26]. A non-unital $\mathrm{C}^{*}$-algebra $A$ is said to be $\sigma$-unital whenever it has a countable approximate unit; in particular every separable $\mathrm{C}^{*}$-algebra is $\sigma$-unital (see, e.g. [14]). If $A$ has real rank zero and is $\sigma$-unital, then it has an approximate unit consisting of an increasing sequence of projections $[6,2.9]$. In fact, if $A$ is a $\sigma$-unital $\mathrm{C}^{*}$-algebra with real rank zero, then, for any projection $P \in \mathcal{M}(A)$, we have that $P A P$ is a $\sigma$-unital $\mathrm{C}^{*}$-algebra with real rank zero, and it has an approximate unit consisting of an increasing sequence of projections; moreover, given projections $p \in A$ and $P \in \mathcal{M}(A)$, if $\left\{p_{n}\right\}$ is an approximate unit of $P A P$ consisting of an increasing sequence of projections, then $p \lesssim P$ if and only if $p \lesssim p_{n}$ for some $n \geq 1$ [13, Lemma 1.3]. Thus, if $A$ is a $\sigma$-unital $\mathrm{C}^{*}$-algebra with real rank zero and stable rank one, and $P \in \mathcal{M}(A) \backslash A$ is a projection with $\left\{p_{n}\right\}$ an approximate unit of $P A P$ consisting of an increasing sequence of projections, we define $\Theta([P])=\left\{[p] \in V(A) \mid p\right.$ is a projection in $\left.P M_{\infty}(A) P\right\}=\{[p] \in$ $V(A) \mid[p] \leq\left[p_{n}\right]$ for some $\left.n \in \mathbb{N}\right\}$. Then, $\Theta([P])$ is a countably generated soft interval in $V(A)$, and moreover, if $D(A)=\Theta\left(\left[1_{\mathcal{M}(A)}\right]\right)$, then the map

$$
\Theta:\left(V(\mathcal{M}(A)),\left[1_{\mathcal{M}(A)}\right]\right) \rightarrow W_{\sigma}^{D(A)}(V(A))
$$

is a normalized monoid isomorphism [13, Section 1], [19, Section 2].

A ring $R$ is said to be (von Neumann) regular provided that, for every $x \in R$, there exists $y \in R$ such that $x y x=x$. We say that a regular ring $R$ has countable unit if there exists an increasing sequence of idempotents $\left\{e_{n}\right\}$ such that $R=\bigcup_{n \geq 1} e_{n} R e_{n}$; such a sequence $\left\{e_{n}\right\}$ is called a countable unit; in particular every countable regular ring has countable unit [3, Section 1]. In fact, if $R$ is a regular ring with countable unit, then, for any idempotent $E \in \mathcal{M}(R)$, we have that $E R E$ is a regular ring with countable unit; moreover, given idempotents $e \in R$ and $E \in \mathcal{M}(R)$, if $\left\{e_{n}\right\}$ is a countable unit of $E R E$, then $e \lesssim E$ if and only if $e \lesssim e_{n}$ for some $n \geq 1$ [3, Lemma 2.1]. Thus, if $R$ is a regular ring with stable rank one and countable unit, and $E \in \mathcal{M}(R) \backslash R$ is an idempotent with $\left\{e_{n}\right\}$ a countable unit of $E R E$, we define $\Theta([E])=\left\{[e] \in V(R) \mid e\right.$ is an idempotent in $\left.E M_{\infty}(R) E\right\}=\{[e] \in$ $V(R) \mid[e] \leq\left[e_{n}\right]$ for some $\left.n \in \mathbb{N}\right\}$. Then, $\Theta([E])$ is a countably generated soft interval in $V(R)$, and moreover, if $D(R)=\Theta\left(\left[1_{\mathcal{M}(R)}\right]\right)$, then the map 


$$
\Theta:\left(V(\mathcal{M}(R)),\left[1_{\mathcal{M}(R)}\right]\right) \rightarrow W_{\sigma}^{D(R)}(V(R))
$$

is a normalized monoid isomorphism [3, Section 2].

Thus, the results in previous sections apply for $R$ any (separable) $\sigma$-unital, non-unital, nonelementary, simple $\mathrm{C}^{*}$-algebra with real rank zero and stable rank one, or for any (countable) non-unital, non-artinian, simple von Neumann regular ring of stable rank one with countable unit, since in both cases it is well-known that the monoid $V(R)$ is a (countable) cancellative, non-atomic, simple, refinement monoid. Also, if $d=\rho(D(R))$ and $u=[e]$ for any non-zero idempotent $e \in R$, then by composing the map $\varphi$ defined in Theorem 3.1 with the map defined above, we get a normalized monoid morphism

$$
\Phi:\left(V(\mathcal{M}(R)),\left[1_{\mathcal{M}(R)}\right]\right) \rightarrow\left(V(R) \sqcup W_{\sigma}^{d}\left(S_{u}\right), d\right)
$$

which is an isomorphism if $V(R)$ is strictly unperforated [19, Theorem 3.9], [3, Theorem 2.11].

In order to simplify the notation, throughout this section we will say that a ring $R$ lies in the class $\mathcal{N}$ if it is a non-unital, non-artinian, simple von Neumann regular ring of stable rank one with countable unit; similarly, we will say that a $\mathrm{C}^{*}$-algebra $A$ lies in the class $\mathcal{N}^{*}$ if it is a $\sigma$-unital, non-unital, non-elementary, simple $\mathrm{C}^{*}$-algebra with real rank zero and stable rank one.

Given $R$ a ring in the class $\mathcal{N}$, or a $\mathrm{C}^{*}$-algebra in the class $\mathcal{N}^{*}$, we can define a new relation between idempotents in $\mathcal{M}(R)$ through the isomorphisms defined in (1) and (2), as follows: given $P, Q \in \mathcal{M}(R)$, we say that $P \precsim Q$ if $\Theta([P]) \subseteq \Theta([Q])$. Notice that, if $p, q \in R$ are idempotents, then $p \precsim q$ if and only if $p \lesssim q$.

Let $R$ be a countable ring $R$ in the class $\mathcal{N}$, or a separable $\mathrm{C}^{*}$-algebra in the class $\mathcal{N}^{*}$ such that $D(R)$ is a functionally complete interval. Given an idempotent $P \in \mathcal{M}(R)$, we say that $P$ is functionally complete if $\Theta([P])$ is a functionally complete interval; in particular, $1_{\mathcal{M}(R)}$ is a functionally complete idempotent if and only if $D(R)$ is a functionally complete interval. Also, given an idempotent $P \in \mathcal{M}(R)$, we have that $\rho^{\prime} \rho(\Theta([P])) \in W_{\sigma}^{D(R)}(V(R))$, so that there exists an idempotent $Q \in \mathcal{M}(R)$ with $\Theta([Q])=\rho^{\prime} \rho(\Theta([P]))$. We denote such an idempotent $Q$ by $P^{c}$. Since for any idempotents $Q_{1}, Q_{2} \in \mathcal{M}(R)$, we have $\Theta\left(\left[Q_{1}\right]\right)=\Theta\left(\left[Q_{2}\right]\right)$ if and only if $Q_{1} \sim Q_{2}$, notice that $P^{c}$ is determined up to equivalence. Certainly $P^{c}$ is functionally complete. Moreover, it is clear that $P$ is functionally complete if and only if $P \sim P^{c}$, and in particular, for any idempotent $P$ in $\mathcal{M}(R), \Phi([P])=\Phi\left(\left[P^{c}\right]\right)$. Under the same hypotheses on $R$, if $e \in R$ is an idempotent, then there exists an idempotent $E \in \mathcal{M}(R)$ such that $\Theta([E])=[0,[e])$. As above, the idempotent $E$ is determined up to equivalence, and we denote it by $\mathcal{O}(e)$.

Thus, using the results in previous sections, we get the following results, that state the existence of some special pathological idempotents in multiplier algebras.

Proposition 4.1. Let $R$ be a countable ring $R$ in the class $\mathcal{N}$, or a separable $C^{*}$-algebra in the class $\mathcal{N}^{*}$ such that $1_{\mathcal{M}(R)}$ is functionally complete, and let $e \in R, E, F \in \mathcal{M}(R)$ be non-zero idempotents. Then:

(1) $\mathcal{O}(e) \precsim e$, and is the biggest idempotent (up to equivalence) in $\mathcal{M}(R)$ satisfying this property.

(2) $E \precsim E^{c}$, and if $\left[E^{c}\right]$ is stably finite, then $E \lesssim E^{c}$ if and only if $E \sim E^{c}$. 
(3) $\left(E^{c}\right)^{c} \sim E^{c}$, and $E \oplus F^{c} \sim E^{c} \oplus F^{c} \sim(E \oplus F)^{c}$.

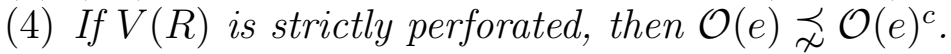

Proof. Notice that countability hypothesis on $V(R)$ is required to guarantee that $\mathcal{O}(e) \in$ $\mathcal{M}(R)$, and $1_{\mathcal{M}(R)}$ functionally complete is required to guarantee that $P^{c} \in \mathcal{M}(R)$ for any idempotent $P \in \mathcal{M}(R)$.

(1) It is Remark 1.6.

(2) The first part is immediate. The second one is an immediate consequence of Corollary 2.4 .

(3) The first part is immediate. The second one is Proposition 2.3.

(4) It is Corollary 2.8.

Also, as a consequence of Proposition 3.8, we have the following result about divisibility of idempotents

Proposition 4.2. Let $R$ be a ring $R$ in the class $\mathcal{N}$, or a $C^{*}$-algebra in the class $\mathcal{N}^{*}$, and let $E \in \mathcal{M}(R) \backslash R$ be an idempotent. Then, for every $n \in \mathbb{N}$ there exists an idempotent $E_{n} \in \mathcal{M}(R) \backslash R$ such that $E \sim n \cdot E_{n}$.

In the case of $\mathrm{C}^{*}$-algebras, Proposition 4.2 is [35, Theorem I (ii)]. For the case of von Neumann regular rings, this result is not known, as far as we know. Now, as a corollary of Theorem 3.2, we get the following result.

Theorem 4.3. Let $R$ be a countable ring $R$ in the class $\mathcal{N}$, or a separable $C^{*}$-algebra in the class $\mathcal{N}^{*}$, let $u \in V(R)^{*}$, and let $d=\Phi\left(\left[1_{\mathcal{M}(R)}\right]\right)$. Then, the following conditions are equivalent:

(1) $V(R)$ is strictly unperforated.

(2) Every idempotent in $\mathcal{M}(R) \backslash R$ is functionally complete.

(3) The map $\Phi:\left(V(\mathcal{M}(R)),\left[1_{\mathcal{M}(R)}\right]\right) \rightarrow\left(V(R) \sqcup W_{\sigma}^{d}\left(S_{u}\right), d\right)$ is an injective monoid morphism.

(4) The subsemigroup of $V(\mathcal{M}(R))$ consisting of equivalence classes of idempotents $E \in$ $\mathcal{M}(R) \backslash R$ such that $\rho(\Theta([E])) \in \mathcal{E}_{\text {fin }}$ is strongly separative.

(5) For any non-zero idempotent $e \in R, \mathcal{O}(e)$ is functionally complete.

Now, as a corollary of Theorem 3.5, we get the following result.

Theorem 4.4. Let $R$ be a ring in the class $\mathcal{N}$, or a $C^{*}$-algebra in the class $\mathcal{N}^{*}$, let $u \in V(R)^{*}$, and let $d=\Phi\left(\left[1_{\mathcal{M}(R)}\right]\right)$. If $1_{\mathcal{M}(R)}$ is functionally complete, then the map

$$
\Phi:\left(V(\mathcal{M}(R)),\left[1_{\mathcal{M}(R)}\right]\right) \rightarrow\left(V(R) \sqcup W_{\sigma}^{d}\left(S_{u}\right), d\right)
$$

is a normalized monoid epimorphism.

There is an interesting case of application for Theorem 4.4. Recall that a $\mathrm{C}^{*}$-algebra $A$ is said to be stable provided that $A \cong A \otimes \mathcal{K}$, where $\mathcal{K}$ denotes the algebra of compact operators over a countable-dimensional Hilbert space. In the case of rings, we say that a ring $R$ is stable provided that $R \cong M_{\infty}(R)$. Notice that, if $A$ is a $C^{*}$-algebra, then $A \otimes \mathcal{K}$ is isomorphic to the norm completion of the pre- $\mathrm{C}^{*}$-algebra $M_{\infty}(A)$.

Lemma 4.5. If $R$ is a stable ring in the class $\mathcal{N}$, or a stable $C^{*}$-algebra in the class $\mathcal{N}^{*}$, then $1_{\mathcal{M}(R)}$ is functionally complete. 
Proof. Since $R$ is stable, we have a countable unit $\left\{e_{n}\right\}$ consisting on an increasing sequence of idempotents such that, for every $n \geq 1$, all the idempotents $e_{n+1}-e_{n}$ are equivalent to a fixed non-zero projection $p \in R$. Then, $\Theta\left(\left[1_{\mathcal{M}(R)}\right]\right)=\{x \in V(R) \mid x \leq n[p]$ for some $n \geq 1\}$, and since $R$ is simple, we conclude that $\Theta\left(\left[1_{\mathcal{M}(R)}\right]\right)=V(R)$.

Hence, we get the following result.

Corollary 4.6. Let $R$ be a stable ring in the class $\mathcal{N}$, or a stable $C^{*}$-algebra in the class $\mathcal{N}^{*}$. Let $u \in V(R)^{*}$, and let $d=\Phi\left(\left[1_{\mathcal{M}(R)}\right]\right)$. Then the map

$$
\Phi:\left(V(\mathcal{M}(R)),\left[1_{\mathcal{M}(R)}\right]\right) \rightarrow\left(V(R) \sqcup W_{\sigma}^{d}\left(S_{u}\right), d\right)
$$

is a normalized monoid epimorphism. Moreover, if $V(R)$ is strictly perforated, then $\Phi$ fails to be injective.

Notice that, if $A$ is a $\sigma$-unital $\mathrm{C}^{*}$-algebra of real rank zero, then the lattice of closed ideals of $\mathcal{M}(A)$ is isomorphic to the lattice of order-ideals of $V(\mathcal{M}(A))$ [34, Theorem 2.3]. The same result is true for a $\sigma$-unital von Neumann regular ring, because of [3, Theorem 2.7]. Hence, in view of Remark 3.7(2), even if injectivity is lost in the strictly perforated case, we still could study the structure of the lattice of (closed) ideals of $\mathcal{M}(A)$ using the techniques developed by Perera [19], provided we could state the arithmetical properties of the set $\Phi^{-1}(f)$ for any $f \in W_{\sigma}^{d}\left(S_{u}\right)$. Thus, to extend the results of Perera to this context, the following question should be answered.

Problem 4.7. Let $M$ be a (countable) conical, cancellative, non-atomic, strictly perforated, simple refinement monoid, let $u \in M$ be a non-zero element, let $D$ be a non-zero functionally complete soft interval, and let $d=\rho(D)$. Describe $\varphi^{-1}(f)$ for every function $f \in W_{\sigma}^{d}\left(S_{u}\right)$.

In the same line, and in order to extend the scope of the results obtained in this paper, there are two questions that should be answered.

Problem 4.8. Let $M$ be a conical, cancellative, non-atomic, simple refinement monoid, and let $D$ be a countably generated soft interval. Is then $\rho^{\prime} \rho(D)$ a countably generated interval?

Problem 4.9. Can we eliminate the hypothesis " $M$ countable" in Proposition 1.5 in order to get $[0, x)$ countably generated?

An affirmative answer to this question would imply that results about injectivity are also true for arbitrary $\mathrm{C}^{*}$-algebras of real rank zero or von Neumann regular rings.

\section{ACKNOWLEDGMENTS}

We want to thank Ken Goodearl and Francesc Perera, who read the preliminary version of this paper, discovered several misprints and oversights, and suggested useful improvements. In addition, we are extremely indebted to the referee for the careful and thoughtful review of this paper.

\section{REFERENCES}

[1] P. Ara, K.R. Goodearl, K.C. O'Meara, E. Pardo, Separative cancellation for projective modules over exchange rings, Israel J. Math. 105 (1998), 105-137. 
[2] P. Ara, E. Pardo, F. Perera, The structure of countably generated projective modules over regular rings, J. Algebra 226 (2000), 161-190.

[3] P. Ara, F. Perera, Multipliers of von Neumann regular rings, Comm. Algebra 28(7) (2000), 33593385.

[4] B. Blackadar, "K-Theory for Operator Algebras", Second Edition, M.S.R.I. Publications 5, Cambridge Univ. Press, Cambridge, 1998.

[5] B. Blackadar, Rational C*-algebras and nonstable K-Theory, Rocky Mountain J. Math. 20 (1990), 285-316.

[6] L.G. Brown, G.K. Pedersen, $C^{*}$-algebras of real rank zero, J. Funct. Anal. 99 (1991), 131-149.

[7] G. Elliott, J. Villadsen, Perforated ordered $K_{0}$-groups, Canad. J. Math. 52(6) (2000), $1164-1191$.

[8] E.G. Evans, Krull-Schmidt and cancellation over local rings, Pacific. J. Math. 46 (1973), $115-121$.

[9] K.R. Goodearl, "Von Neumann Regular Rings", Pitman, London, 1979; "," Second Ed., Krieger, Malabar, Fl., 1991.

[10] K.R. Goodearl, "Partially Ordered Abelian Groups with Interpolation", Math. Surveys and Monographs 20, A.M.S., Providence, 1986.

[11] K.R. Goodearl, D.E. Handelman, Stenosis in dimension groups and AF-C*-algebras, J. reine angew. Math. 332 (1982), 1-98.

[12] K.R. GoodeArL, Extensions of dimension groups and $\mathrm{AF} \mathrm{C}^{*}$-algebras, J. reine angew. Math. 412 (1990), 150-219.

[13] K.R. Goodearl, $K_{0}$ of multiplier algebras of $\mathrm{C}^{*}$-algebras with real rank zero, K-Theory 10 (1996), 419-489.

[14] G.J. Murphy, "C*-algebras and Operator Theory", Academic Press, Boston, 1990.

[15] E. PArdo, On the representation of simple Riesz groups, Comm. Algebra 26(1) (1998), 155-170.

[16] E. Pardo, Metric completions of ordered groups and $K_{0}$ of exchange rings, Trans. Amer. Math. Soc. 350 (1998), 913-933.

[17] E. PARdo, Finite projections in multiplier algebras, Rocky Mountain J. Math. 31(2) (2001), 581-594.

[18] E. PARDo, Embedding rank one simple groups into rank one simple Riesz groups, Glasgow Math. J. (to appear)

[19] F. PERERA, Ideal structure of multiplier algebras of simple $\mathrm{C}^{*}$-algebras with real rank zero, Canad. J. Math. 53(3) (2001), 592-630.

[20] M. Rørdam, On sums of finite projections, "Operator Algebras and Operator Theory" (ed. L. Ge, H. Lin, and S. Zhang), Contemp. Math. 228 (1998), 327-340.

[21] M. RøRdam, J. Villadsen, On the ordered $K_{0}$-group of universal free product $\mathrm{C}^{*}$-algebras, $K$-Theory 15 (1998), 307-322.

[22] A. Toms, Strongly perforated $K_{0}$-groups of simple $\mathrm{C}^{*}$-algebras, Canad. Math. Bull. (to appear).

[23] L.N. VAserstein, Stable rank of rings and dimensionality of topological spaces, Funct. Anal. Appl. 5 (1971), 102-110.

[24] L.N. Vaserstein, Bass' first stable rank condition, J. Pure Appl. Algebra 34 (1984), 319-330.

[25] J. Villadsen, Simple C*-algebras with perforation, J. Funct. Anal. 154 (1998), 110-116.

[26] R.B. WARfield, JR., Exchange rings and decompositions of modules, Math. Ann. 199 (1972), 31-36.

[27] N.E. Wegge-Olsen, "K-Theory and $C^{*}$-algebras", Oxford Univ. Press, Oxford, 1993.

[28] F. Wehrung, Injective positively ordered monoids I, J. Pure Appl. Algebra 83 (1992), 43-82.

[29] F. Wehrung, Restricted injectivity, transfer property and decompositions of separative positively ordered monoids, Comm. Algebra 22(5) (1994), 1747-1781.

[30] F. Wehrung, The universal theory of ordered equidecomposability types semigroups, Canad. J. Math. 46(5) (1994), 1093-1120.

[31] F. Wehrung, Norm-closed intervals of norm-complete ordered abelian groups, Positivity 1 (1997), 271-290.

[32] F. Wehrung, Embedding simple commutative monoids into simple refinement monoids, Semigroup Forum 56 (1998), 104-129.

[33] S. Zhang, Diagonalizing projections in multiplier algebras and in matrices over a C*-algebra, Pacif. J. Math. 145 (1990), 181-200. 
[34] S. Zhang, A Riesz decomposition property and ideal structure of multiplier algebras, J. Operator Theory 24 (1990), 209-225.

[35] S. Zhang, Matricial structure and homotopy type of simple $\mathrm{C}^{*}$-algebras with real rank zero, J. Operator Theory 26 (1991), 283-312.

Departamento de Matemáticas, Universidad de Cádiz, Apartado 40, 11510 Puerto Real (CÁDIZ), SPAIN.

E-mail address: francisco.ortus@uca.es, enrique.pardo@uca.es 